\title{
Selective growth of a-form zinc phthalocyanine nanowire crystals via the flow rate control of physical vapor transport
}

\author{
Youngkwan Yoon ${ }^{1}$, Soyoung $\mathrm{Kim}^{1}$ and Hee Cheul Choi ${ }^{1}$
}

\begin{abstract}
a-Form zinc phthalocyanine ( $\mathrm{ZnPc})$, a kinetically favorable form of $\mathrm{ZnPc}$, is an efficient photosensitizer for photodynamic cancer therapy, especially when it is formed into nanowires (NWs), which have high water dispersibility. To take full advantage of this material, it is necessary to selectively grow kinetically favorable a-form ZnPc NWs over thermodynamically favorable $\beta$-form ZnPc NWs. The present study reports the selective growth of kinetically favorable a-form ZnPc NW crystals by a physical vapor transport method with flow rate control of the carrier gas. The flow rate is key for the dominant growth of a-form ZnPc NWs, thus enabling control of the crystal size, which critically affects the stability of ZnPc crystals. The yield of a-form ZnPc NWs was over $98 \%$ at a flow rate of $2000 \mathrm{sccm}$ of Ar carrier gas. This approach could be applied for various Pc molecules, including copper phthalocyanine (CuPc). Our findings might contribute to the development of novel strategies for the selective growth of high-quality kinetically favorable crystals with a specific structure and provide great opportunities for their various practical applications.
\end{abstract}

\section{Introduction}

Kinetically favorable metastable crystals frequently exhibit remarkable properties superior to those of their thermodynamically favorable and more stable products. ${ }^{1-7}$ Therefore, the selective growth of the desired crystals, especially in kinetically favorable structures, is an important issue not only for the fundamental study of the crystal growth mechanism but also for their practical applications. However, such specific crystal structure control is difficult because it is normally determined by thermodynamic equilibrium and influenced by various crystallization conditions that affect not only thermodynamic but also kinetic controls. ${ }^{4,8-12}$ Therefore, polymorphism is quite commonly observed, especially in organic and organometallic molecular crystals, due to their various weak nondirectional intermolecular van der Waals forces. ${ }^{4,13-15}$ Polymorph crystals having kinetically and thermodynamically favorable structures show

\footnotetext{
Correspondence: Hee Cheul Choi (choihc@postech.edu)

${ }^{1}$ Department of Chemistry, Pohang University of Science and Technology

(POSTECH), 77 Cheongam-ro, Nam-gu, Pohang-si 37673, Korea
}

significantly different properties depending on the stacking structure. For example, the metastable $\alpha$-phase of 2,6dichloronaphthalene diimide exhibits much higher mobility than the stable $\beta$-phase due to their different electronic couplings between neighboring molecules. ${ }^{4,5}$ Additionally, metastable Y-form titanyl phthalocyanine shows a photogeneration quantum yield greater than $90 \%$, which is much higher than those of the stable $\alpha$ - and $\beta$-forms. ${ }^{2,6,7}$ To date, there have been many trials to control the crystal morphology and structure via various strategies without suggestion of a facile strategy for obtaining such kinetically favorable structure-controlled crystals.

Previously, our group reported $\alpha$-form ZnPc nanowires (NWs) that showed substantially improved water dispersibility and highly efficient dual photodynamic and photothermal performances, both of which have a synergistic effect in cancer phototherapy, as demonstrated by both in vitro and in vivo tests. ${ }^{3}$ However, the difficult phase separation of simultaneously obtained kinetically favorable $\alpha$-form and thermodynamically favorable 
$\beta$-form ZnPcs still remains a major limitation in the practical usage of this material as an anticancer agent because the presence of a small amount of $\beta$-form ZnPc causes aggregation and precipitation in solution. Therefore, the selective growth of high-quality and high-yield kinetically favorable $\alpha$-form ZnPc NWs is an important and urgent issue for practical applications, including clinical tests. Herein, we focused on the development of a facile and efficient method for the selective growth of $\alpha$ form $\mathrm{ZnPc}$ NWs and found that the flow rate control of carrier gas during the physical vapor transport (PVT) process could induce the dominant growth of kinetically favorable $\alpha$-form ZnPc NWs. From various studies on Pc, it is well known that the crystal phase is highly related to the crystal size regardless of the growth method. ${ }^{16-20}$ Control of the crystal size during solution-phase crystallization is difficult without using additional chemicals such as capping reagents and surfactants. Therefore, we used the PVT method, a vapor-phase crystallization method that is appropriate to obtain pure and highquality crystals. By increasing the flow rate of the carrier gas, we successfully reduced the crystal size of $\mathrm{ZnPc} \mathrm{NWs}$ and stabilized the $\alpha$-form ZnPc NW crystals, which enabled the growth of high-yield $\alpha$-form ZnPc NWs very selectively. In particular, the $\alpha$-form ZnPc NWs grown at a carrier gas flow rate of $2000 \mathrm{sccm}$ show extremely high water dispersibility without any significant aggregation after dispersion in water for $8 \mathrm{~h}$.

\section{Materials and methods}

\section{Synthesis of ZnPc and CuPc NWs}

$\mathrm{ZnPc}$ NWs and CuPc NWs were prepared using commercially available zinc phthalocyanine (ZnPc, 97\%, Sigma-Aldrich) and copper phthalocyanine (CuPc, 97\%, Sigma-Aldrich) precursors, respectively, without further purification. Approximately $20 \mathrm{mg}$ of precursor loaded in a ceramic boat was placed at the center of a tube furnace using a quartz protection tube. We placed a piece of $\mathrm{Si}$ (100) substrate at the end region of the quartz tube where the temperature naturally decreased to less than $80^{\circ} \mathrm{C}$ to collect the resulting crystals effectively. After flushing the quartz tube using Ar gas at a flow rate of $1000 \mathrm{sccm}$ for $5 \mathrm{~min}$, we increased the furnace temperature up to $500^{\circ} \mathrm{C}$ under specific Ar flow rates (50, 200, 800, $2000 \mathrm{sccm}$ ). After $10 \mathrm{~min}$ of reaction at the target temperature, the furnace was cooled naturally to room temperature by turning the power of the furnace off.

\section{Characterization of $\mathrm{ZnPc}$ and CuPc NWs}

The morphology of the resulting crystals was analyzed by scanning electron microscopy (SEM, JSM-7401F, JEOL). To prevent electron charging of the crystals, a platinum coating was applied to create a conductive layer on the crystal surface. Additionally, X-ray diffraction patterns of $\mathrm{ZnPc}$ and $\mathrm{CuPc}$ crystals were obtained from the 5D beamline at the Pohang Accelerator Laboratory (PAL). All obtained data were converted to the wavelength of $\mathrm{CuK \alpha}(\lambda(\mathrm{K} 1.541841 \AA)$ for better comparison with references. High-resolution transmission electron microscopy (HRTEM, JEM-2200FS, JEOL) was performed for structure analysis, and the samples for TEM measurements were prepared by stamping the $\mathrm{ZnPc}$ crystals onto a carbon-coated $\mathrm{Cu}$ grid.

\section{Preparation and quantitative analysis of dispersed solutions}

For the quantitative analysis of $\alpha$-form $\mathrm{ZnPc}$ crystals obtained at different flow rates, we used a standard calibration method using distinctive light absorption bands of $\alpha$-form ZnPc measured by a UV-VIS spectrometer (UV2600, SHIMADZU). To obtain a standard calibration curve between the concentration and absorbance of $\alpha$ form $\mathrm{ZnPc}$, only $\alpha$-form $\mathrm{ZnPc}$ dispersed aqueous solutions were prepared as follows:

$\mathrm{ZnPc} \mathrm{NW}$ dispersed aqueous solutions were prepared by dissolving ZnPc crystals obtained by the PVT method in deionized (DI) water, followed by $40 \mathrm{~min}$ of sonication (Fig. S1a). After $24 \mathrm{~h}$, the upper (well-dispersed $\alpha$-form) part of the ZnPc NW solution was isolated, and $\alpha$-form $\mathrm{ZnPc}$ was obtained in powder form by vacuum drying of the isolated solution. Using powder $\mathrm{X}$-ray diffraction (PXRD) and UV-VIS results, we confirmed that the resulting product was $\alpha$-form ZnPc (Fig. S1c and d). The obtained pure $\alpha$-form ZnPc was redispersed in water and sequentially diluted to obtain reference solutions with five different $\alpha$-form ZnPc concentrations (Fig. S1b). The absorption intensity of the reference solutions increased according to the $\alpha$-form ZnPc concentration (Fig. S1d). The concentration and absorbance showed a linear relationship, as shown in Fig. S1e.

Based on these reference data, the concentration of $\alpha$ form $\mathrm{ZnPc}$ in each solution obtained at different flow rates was determined by measuring the optical absorbance at $785 \mathrm{~nm}$ of the upper (well-dispersed) part of the 8-hstored $\mathrm{ZnPc}$ solution. By matching the absorbance of the solution of interest with the reference linear fitting data represented in Fig. S1e, the weight percent of $\alpha$-form $\mathrm{ZnPc}$ in each flow rate condition was calculated by multiplying the concentration of $\alpha$-form $\mathrm{ZnPc}$ by the volume of water.

\section{Results and discussion}

To obtain high-yield and high-quality $\alpha$-form $\mathrm{ZnPc}$ NWs, we used the PVT method, which employs vaporization, condensation and recrystallization processes of target precursor molecules. ${ }^{10,21}$ Briefly, ZnPc powder located in the center of a tube furnace was vaporized at $500{ }^{\circ} \mathrm{C}$, and Ar gas was used as a carrier gas to deliver 


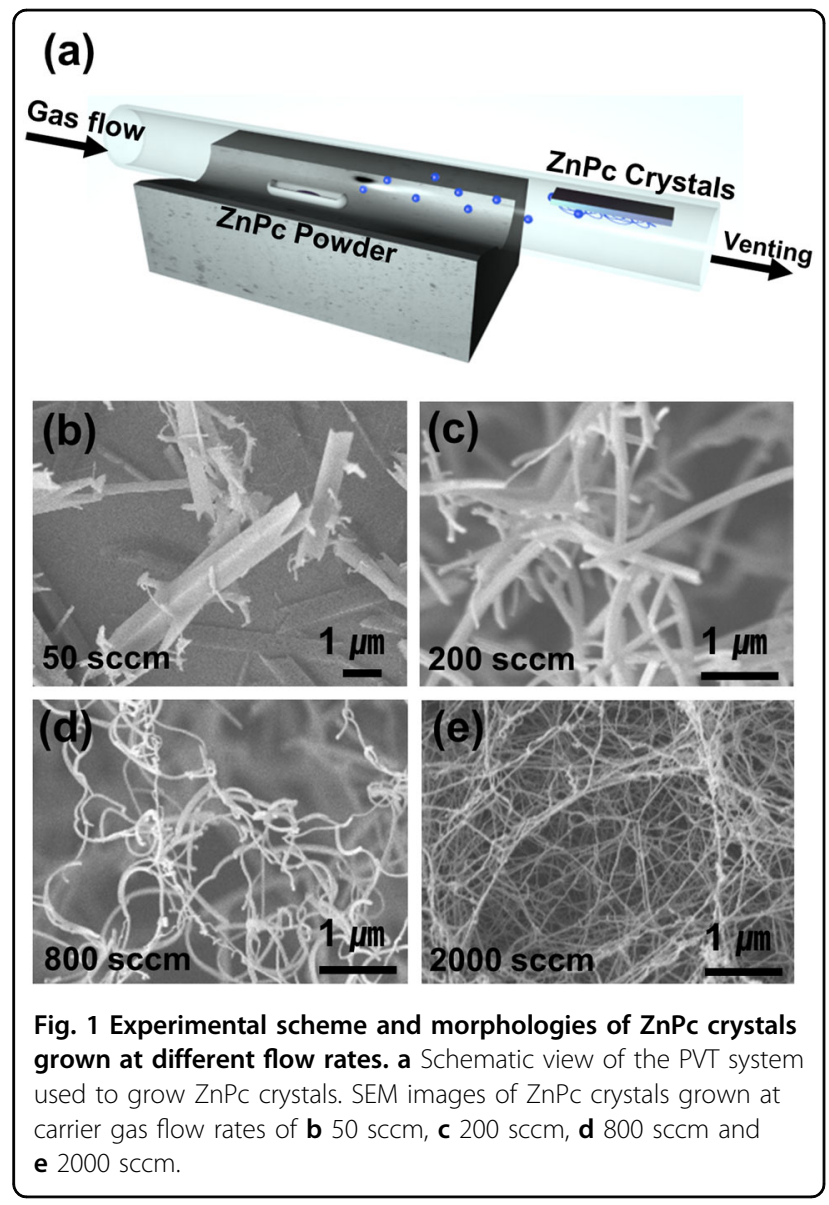

ZnPc vapors to a cooler region. A silicon substrate was placed at the end region of the furnace where the temperature naturally decreased to collect resulting crystals efficiently (Fig. 1a). After $10 \mathrm{~min}$ of reaction, blue ZnPc crystals were formed at the end of the tube furnace.

The preferred formation of $\alpha$ - and $\beta$-form $\mathrm{ZnPc}$ crystals depends on the crystal size due to their lattice potentials and surface energies. ${ }^{18}$ According to the Buckingham equation used for estimating the interatomic nonbonding potentials in organic molecular crystals, $\alpha$-form $\mathrm{ZnPc}$ is more stable than the $\beta$-form when the crystal size is small. ${ }^{18}$ Normally, in the case of the vapor-phase deposition process, the crystal size is highly affected by the flow rate of the carrier gas. ${ }^{21,22}$ Therefore, we varied the flow rate of Ar gas from 50 to $2000 \mathrm{sccm}$ to evaluate the effect of carrier gas flow rate on the selection of the $\mathrm{ZnPc}$ structure. Figure 1b-e shows SEM images of ZnPc crystals obtained at different flow rates of Ar gas. As the flow rate increased, the width of the crystals decreased from approximately $500 \mathrm{~nm}$ (at $50 \mathrm{sccm}$ (Fig. 1b)) to $50 \mathrm{~nm}$ (at $2000 \mathrm{sccm}$ (Fig. 1e)). To confirm the crystal structures, we performed the PXRD and high-resolution transmission electron microscopy (HR-TEM). For clear observation of each diffraction peak, the intensity of the
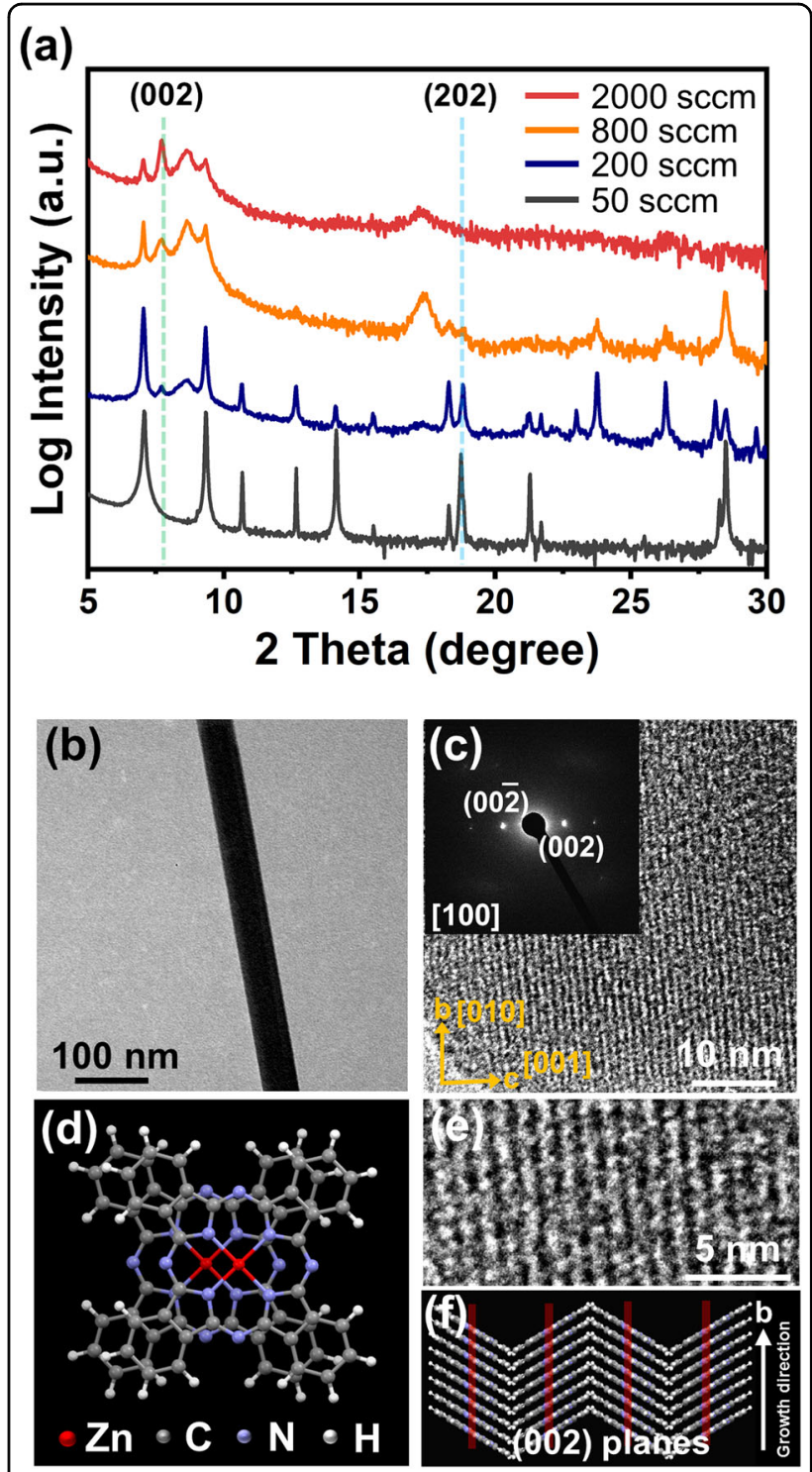

Fig. 2 Crystal structure analysis of a-form ZnPc NW crystals. a PXRD patterns of ZnPc NW crystals grown at different flow rates. b Low-magnification TEM image and $\mathbf{c}$ high-resolution TEM (HR-TEM) image of the a-form ZnPc NW. The inset shows the ED pattern of the ZnPc NW with [100] projection. $\mathbf{d}$ Stacking arrangement of the a-form $\mathrm{ZnPc}$ crystal along the normal of the planar molecule. e Magnified image of $\mathbf{c}$. $\mathbf{f}$ Schematic view of the successive stacking of $\mathrm{ZnPc}$ molecules on the [100] projection.

PXRD patterns represented in Fig. 2a was converted from a linear scale to a logarithmic scale. The PXRD patterns plotted on a linear scale are represented in Fig. S2. Figure 2a shows the PXRD patterns of the resulting crystals grown at different flow rates. Due to the different packing structures of $\alpha$ - and $\beta$-form ZnPc crystals, different characteristic X-ray diffraction patterns were obtained. One of the representative characteristic diffraction planes of the $\alpha$ - and $\beta$-forms of ZnPc crystals are the (002) and 
(202) planes, which show diffraction peaks at $2 \theta=7.712^{\circ}$ and $2 \theta=18.783^{\circ}$, respectively. ${ }^{23-26}$ In the case of $\mathrm{ZnPc}$ crystals grown at a flow rate of $50 \mathrm{sccm}$ (black line in Fig. 2a), a diffraction peak attributed to the (202) plane of the $\beta$-form is clearly observed, while that attributed to the (002) plane of the $\alpha$-form is absent, which implies that $\beta$-form ZnPc crystals are dominantly obtained at relatively low carrier gas flow rates. Furthermore, the (202) diffraction peak of the $\beta$-form gradually decreased while a new (002) peak of the $\alpha$-form appeared as the flow rate increased. At a flow rate of $2000 \mathrm{sccm}$ (red line in Fig. 2a), we observed a clear (002) diffraction peak from the $\alpha$ form and a disappearance of the (202) diffraction peak from the $\beta$-form, which means that $\alpha$-form ZnPc crystals are selectively grown at a relatively high flow rate. These results show that the crystal phase of $\mathrm{ZnPc}$ can be successfully controlled by changing the flow rate of the carrier gas in the PVT method. Furthermore, we tried to analyze several peaks in the XRD data based on the JC-PDS database. The peaks that appeared at $\sim 7.0^{\circ}$ in both $\alpha$ - and $\beta$-form $\mathrm{ZnPc}$ were difficult to distinguish due to the small difference in the two-theta values of the (200) plane of the $\alpha$-form and (101) plane of the $\beta$-form, with peaks appearing at $6.8^{\circ}$ and $7.0^{\circ}$, respectively. ${ }^{23-26}$ Additionally, the peaks appearing at $23.7^{\circ}$ and $26.2^{\circ}$ are consistent with the (211) and (212) planes of $\beta$-form ZnPc, respectively. ${ }^{23-26}$ The absence of two peaks at a flow rate of $50 \mathrm{sccm}$ was due to relatively well-defined and homogeneous exposed crystal facets (Fig. 1b) compared to other ZnPc crystals obtained at flow rates of 200 and $800 \mathrm{sccm}$, of which the exposed planes were inhomogeneous and random due to their cylindrical wire morphology (Fig. 1c, d).

The characteristic crystal planes of $\alpha$ - and $\beta$-form ZnPc crystals were also confirmed using TEM measurements. Figure $2 b$ shows a low-magnification image of an $\alpha$-form $\mathrm{ZnPc}$ NW that has a uniform surface with a width of $\sim 50 \mathrm{~nm}$ and a length of a few micrometers. The width of the thinnest NW obtained at a flow rate of $2000 \mathrm{sccm}$ was $\sim 12 \mathrm{~nm}$ (Fig. S3). Additionally, Fig. 2c and e show HRTEM images of the $\alpha$-form ZnPc NW shown in Fig. $2 b$, which show well-defined crystalline planes. The inset in Fig. 2c shows an electron diffraction (ED) pattern of a NW with clear diffraction spots having a lattice distance of $1.134 \mathrm{~nm}$, which is consistent with the distance between the (002) planes observed in the PXRD pattern in Fig. 2a $\left(\mathrm{d}_{(002)}=11.464 \AA\right.$ at $\left.2 \theta=7.712^{\circ}\right)$. Figure $2 \mathrm{e}$ shows a magnified image of the (002) lattice that corresponds to the aligned $\mathrm{Zn}$ ions, as represented by the red lines in Fig. 2f, which shows growth of ZnPc NWs along the [010] direction stacked by $\pi-\pi$ interactions. Figure $2 d$ shows a molecular structure with a detailed stacking arrangement of $\alpha$-form $\mathrm{ZnPc}$ along the normal of the planar molecule. The largest difference in the molecular packing structure between $\alpha$ - and $\beta$-form $\mathrm{ZnPcs}$ is the angle between the normal of the planar $\mathrm{ZnPc}$ molecule and the column direction (b axis). ${ }^{18}$ Due to the small angle of $\alpha$-form $\mathrm{ZnPc}\left(\approx 25^{\circ}\right)$ compared to the $\beta$-form $\left(\approx 45^{\circ}\right)$, the zinc and nitrogen atoms of $\alpha$-form $\mathrm{ZnPc} \mathrm{NWs}$ have available bonding sites that can interact with water molecules through coordination and hydrogen bonds. ${ }^{3}$ On the other hand, water molecules cannot interact with $\beta$-form $\mathrm{ZnPc}$ because $\mathrm{Zn}(\mathrm{II})$ ions and nitrogen atoms form coordination bonds to adjacent ZnPcs. ${ }^{3}$ To confirm the detailed crystal structure of the $\beta$-form ZnPc NWs, we also obtained HR-TEM images and ED patterns (Fig. S4). The lattice distance of the (202) plane of the $\beta$-form is $0.480 \mathrm{~nm}$, which matches well with our PXRD results $\left(\mathrm{d}_{(202)}=4.724 \AA\right.$ at $\left.2 \theta=18.783^{\circ}\right)$ in Fig. 2a. From the crystal structure analysis, we confirmed the relationship between the crystal phase and carrier gas flow rate, which became the key for the selective growth of $\alpha$-form ZnPc. Additionally, a phase diagram of the $\mathrm{ZnPc} \mathrm{NW}$ indicating its phase transformation according to its size was generated by measuring the crystal size and corresponding phases of the ZnPc NW by TEM analysis (Fig. S5c). The width of ZnPc NWs was determined from the TEM images, and the phase of ZnPc NWs was identified from the ED patterns. The experimentally measured largest width of the $\alpha$-form and the smallest width of the $\beta$-form were $143 \mathrm{~nm}$ and $172 \mathrm{~nm}$, respectively (Fig. S5a and b). Figure 55c displays a phase diagram showing the range of critical size for phase transformation, $140 \sim 170 \mathrm{~nm}$, of which statistical data were obtained by measuring 37 different ZnPc NWs. These results were well matched with a reference paper that suggests the critical size for the phase transformation of $\mathrm{ZnPc}$ films to be 100 $150 \mathrm{~nm}$ experimentally and $200 \sim 300 \mathrm{~nm}$ by calculations. $^{16}$

The most remarkable property of the selectively grown $\alpha$-form $\mathrm{ZnPc} N W s$ is the drastically improved water dispersibility. To confirm the water dispersibility of $\mathrm{ZnPc}$ crystals obtained at different flow rates, the same amount of each product was examined by dispersing the material in DI-water and monitoring any precipitate for a designated time. The top row of Fig. 3a shows a photograph of the solutions obtained after $40 \mathrm{~min}$ of ultrasonication to disperse $\alpha$-form ZnPc NWs uniformly. Except for the leftmost solution prepared using ZnPc crystals obtained at a flow rate of $50 \mathrm{sccm}$, which is pale blue, the other solutions show a similar darker blue color. After $8 \mathrm{~h}$ of storage under ambient conditions, the $\mathrm{ZnPc}$ solution of the crystals obtained at a flow rate of $50 \mathrm{sccm}$ shows severe precipitation, while as the flow rate increases, the precipitation decreases significantly (the bottom row of Fig. 3a). In particular, the solution of the ZnPc crystals obtained at a flow rate of $2000 \mathrm{sccm}$ (rightmost side of Fig. 3b) shows a highly improved water dispersibility 


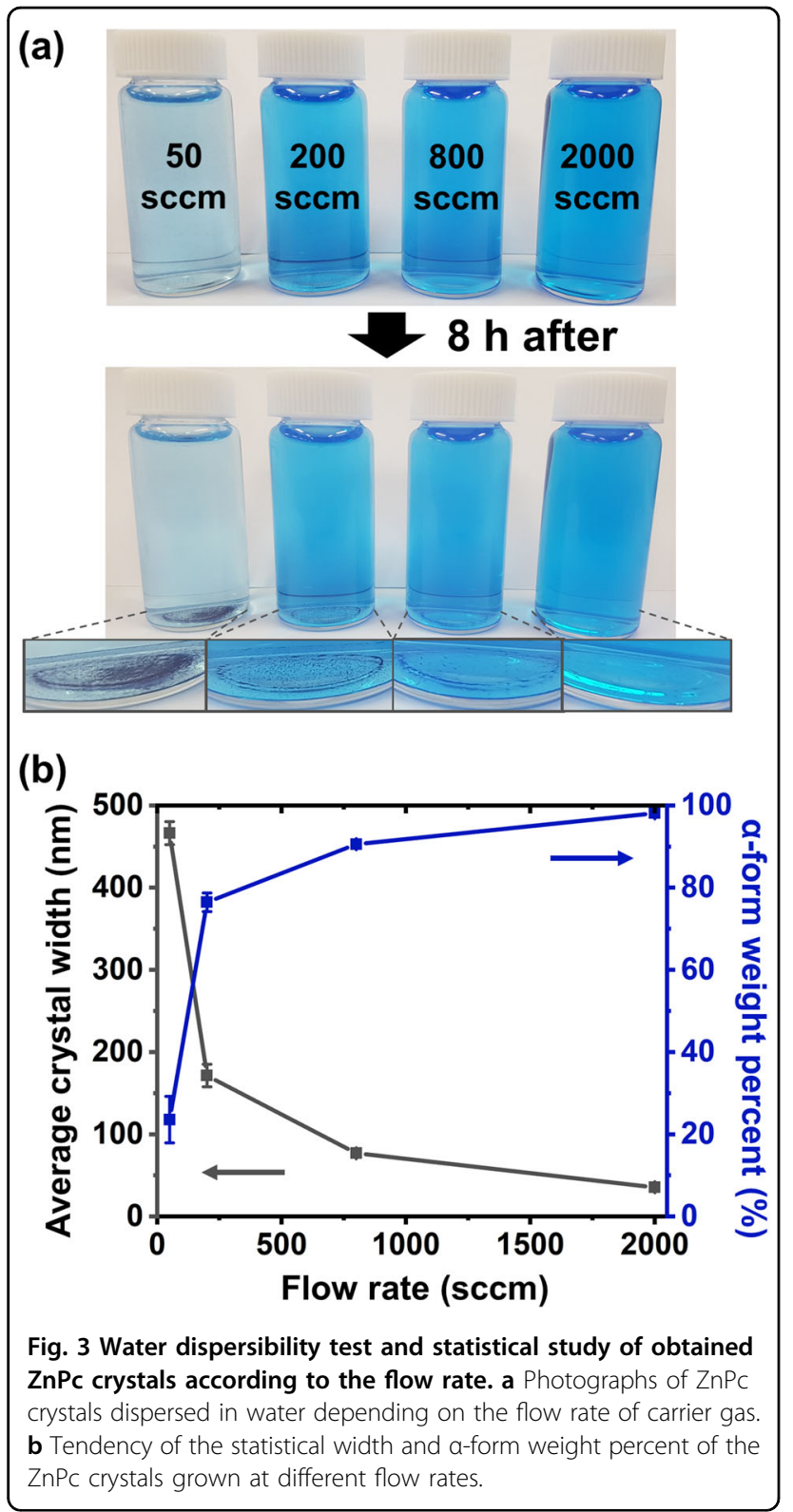

without significant precipitation. These results agree well with the PXRD data that show an increased ratio of $\alpha$ form $\mathrm{ZnPc}$ as the flow rate of the carrier gas increased.

For the quantitative analysis of $\alpha$-form $\mathrm{ZnPc} \mathrm{NW}$ solutions, we obtained UV-VIS spectra of the solutions after removing the precipitates ( $\beta$-form $\mathrm{ZnPc}$ ). Based on the characteristic absorption peak of $\alpha$-form $\mathrm{ZnPc}$ that appeared at $785 \mathrm{~nm}$, the weight percentage of $\alpha$-form $\mathrm{ZnPc}$ in the solution was calculated by comparing the peak intensity with the absorption spectrum of the reference solutions (Fig. S1). As expected from the PXRD data (Fig. 2a), the calculated weight percent of $\alpha$-form $\mathrm{ZnPc}$ in the product increased as the flow rate of the carrier gas increased (blue line in Fig. 3b). Additionally, to our surprise, the percentage of the $\alpha$-form reached over $98 \%$ at a flow rate of $2000 \mathrm{sccm}$. The black line in Fig. 3b shows the relationship between the flow rate and average width of $\mathrm{ZnPc} \mathrm{NW}$ crystals, which decreases as the flow rate increases from $460 \mathrm{~nm}(50 \mathrm{sccm})$ to $35 \mathrm{~nm}$ $(2000 \mathrm{sccm})$. By comparing and analyzing three important values, i.e., average width, weight percent of $\alpha$-form $\mathrm{ZnPc}$ NWs and flow rate of the carrier gas, we clearly confirmed that $\alpha$-form ZnPc NWs were favorably grown at a high carrier gas flow rate, by which the width of the NWs was effectively reduced.

To confirm the versatility of our strategy, we tried to control the phase of copper phthalocyanine $(\mathrm{CuPc})$, which is well known as a good hole injection material for lightemitting diodes. ${ }^{27,28}$ Due to the low solubility of this material in water and other organic solvents, uniform coating of $\mathrm{CuPc}$ on the target substrate is one of the major hurdles for device applications. ${ }^{29,30}$ Therefore, we controlled the flow rate of the carrier gas to reduce the size of the $\mathrm{CuPc}$ crystals to improve their water dispersibility. Similar to $\mathrm{ZnPc}$, CuPc was formed into a nanowireshaped morphology, and the width of the CuPc NWs was successfully reduced by increasing the flow rate of the carrier gas (Fig. S6). When we dispersed the resulting $\mathrm{CuPc}$ NWs obtained at each flow rate condition in water, CuPc NWs grown at a high flow rate exhibited a higher water dispersibility (Fig. S7). Additionally, we verified that the metastable form of $\mathrm{CuPc}$ was dominantly grown at a relatively high flow rate, while the stable $\beta$-form of $\mathrm{CuPc}$ was mainly grown at a relatively low flow rate through PXRD analysis (Fig. S8). From these results, we convince that our flow rate-controlled PVT method would be applicable to various Pc crystals.

\section{Conclusions}

We successfully obtained a high yield of $\alpha$-form $\mathrm{ZnPc}$ NWs that show outstanding water dispersibility without aggregation or significant precipitation. The selective growth of $\alpha$-form ZnPc NWs was achieved by simply controlling the flow rate of the carrier gas in the PVT method. Through morphology observation and crystal structure analysis, we proved that the width of $\mathrm{ZnPc}$ crystals was successfully controlled by changing the flow rate of the carrier gas, which affects the selection of the crystal phase of ZnPc. Through UV-VIS analysis, we confirmed that more than $98 \%$ of the ZnPc crystals were $\alpha$-form ZnPc. Our findings proved that flow rate control during the PVT process could be an effective way to obtain kinetically favorable $\mathrm{ZnPc}$ crystals with a specific phase. We believe that our results will contribute to the understanding of the crystallization of molecules, especially in terms of crystal structure control, and offer a great opportunity for various Pcs for their practical applications in aqueous solution form. 


\section{Acknowledgements}

The authors acknowledge funding from the Veteran Researcher Grant (No. 2019R1A2C2004259) managed by the National Research Foundation of Korea (NRF) and Samsung Electronics. For the structure analysis, powder X-ray diffraction patterns were obtained at the 5D beamline of the Pohang Accelerator Laboratory (PAL, South Korea). Morphology and crystal structure observations of ZnPc NWs were carried out using HR-TEM at the National Institute for Nanomaterials Technology (NINT) in Pohang, South Korea.

\section{Conflict of interest}

The authors declare that they have no conflict of interest.

\section{Publisher's note}

Springer Nature remains neutral with regard to jurisdictional claims in published maps and institutional affiliations.

Supplementary information is available for this paper at https://doi.org/ 10.1038/s41427-020-0198-7.

Received: 15 November 2019 Revised: 23 December 2019 Accepted: 25 December 2019.

Published online: 7 February 2020

\section{References}

1. Wang, X., Garcia, T., Monaco, S., Schatschneider, B. \& Marom, N. Effect of crystal packing on the excitonic properties of rubrene polymorphs. CrystEngComm 18, 7353-7362 (2016).

2. Law, K. Y. Organic photoconductive materials: recent trends and developments. Chem. Rev. 93, 449-486 (1993).

3. Moon, H. K. et al. Significant increase in the water dispersibility of zinc phthalocyanine nanowires and applications in cancer phototherapy. NPG Asia Mater. 4, e12 (2012)

4. Zhen, Y. G., Dong, H. L., Jiang, L. \& Hu, W. P. Tailoring crystal polymorphs of organic semiconductors towards high-performance field-effect transistors. Chin. Chem. Lett. 27, 1330-1338 (2016).

5. He, T. et al. Single-crystal field-effect transistors of new Cl2-NDI polymorph processed by sublimation in air. Nat. Commun. 6, 5954 (2015).

6. Li, X., Xiao, Y., Wang, S. \& Li, X. Ultra-photosensitive Y-type titanylphthalocyanine nanocrystals: preparation and photoelectric properties. Dye. Pigment. 125 44-53 (2016).

7. Weiss, D. S. \& Abkowitz, M. Advances in organic photoconductor technology. Chem. Rev. 110, 479-526 (2010).

8. Desiraju, G. R. Cryptic crystallography. Nat. Mater. 1, 77-79 (2002).

9. Zhao, X., Bao, Z., Sun, C. \& Xue, D. Polymorphology formation of Cu2O: a microscopic understanding of single crystal growth from both thermodynamic and kinetic models. J. Cryst. Growth 311, 711-715 (2009).

10. Park, C., Park, J. E. \& Choi, H. C. Crystallization-induced properties from morphology-controlled organic crystals. Acc. Chem. Res. 47, 2353-2364 (2014).

11. Martí-Rujas, J. \& Kawano, M. Kinetic products in coordination networks: Ab initio X-ray powder diffraction analysis. Acc. Chem. Res. 46, 493-505 (2013).
12. Zhang, Z. et al. The impact of interlayer electronic coupling on charge transport in organic semiconductors: a case study on titanylphthalocyanine single crystals. Angew. Chemie Int. 55, 5206-5209 (2016).

13. He, P. et al. Tuning the crystal polymorphs of alkyl thienoacene via solution self-assembly toward air-stable and high-performance organic field-effect transistors. Adv. Mater. 27, 825-830 (2015).

14. Vasseur, K. et al. Correlating the polymorphism of titanyl phthalocyanine thin films with solar cell performance. J. Phys. Chem. Lett. 3, 2395-2400 (2012).

15. Wang, K. et al. Organic polymorphs: one-compound-based crystals with molecular-conformation- and packing-dependent luminescent properties. Adv. Mater. 26, 6168-6173 (2014).

16. Vergnat, C., Landais, V., Legrand, J. F. \& Brinkmann, M. Orienting semiconducting nanocrystals on nanostructured polycarbonate substrates: impact of substrate temperature on polymorphism and in-plane orientation. Macromolecules 44, 3817-3827 (2011).

17. Löbbert, G. in Ullmann's Encycl. Ind. Chem. 27, 181-213 (Wiley-VCH Verlag $\mathrm{GmbH} \&$ Co. KGaA, 2000).

18. Iwatsu, F. Size effects on the alpha-beta transformation of phthalocyanine crystals. J. Phys. Chem. 92, 1678-1681 (1988).

19. Fryer, J. R., McKay, R. B., Mather, R. R. \& Sing, K. S. W. The technological importance of the crystallographic and surface properties of copper phthalocyanine pigments. J. Chem. Technol. Biotechnol. 31, 371-387 (1981).

20. Belenguer, A. M. et al. Understanding the influence of surface solvation and structure on polymorph stability: a combined mechanochemical and theoretical approach. J. Am. Chem. Soc. 140, 17051-17059 (2018).

21. Yoon, S. M. et al. Vaporization-condensation-recrystallization process-mediated synthesis of helical m-aminobenzoic acid nanobelts. Langmuir 23, 11875-11882 (2007)

22. Ullah, A. R., Micolich, A. P., Cochrane, J. W. \& Hamilton, A. R. The effect of temperature and gas flow on the physical vapour growth of mm-scale rubrene crystals for organic FETs. Device Process Technol. Microelectronics MEMS Photonics Nanotechnol. N 6800680005 (2007).

23. Senthilarasu, S. et al. Characterization of zinc phthalocyanine ( $\mathrm{ZnPc}$ ) for photovoltaic applications. Appl. Phys. A Mater. Sci. Process 77, 383-389 (2003).

24. Iwatsu, F. Crystal behavior of zinc phthalocyanine films in alcohols. J. Cryst. Growth 71, 629-638 (1985).

25. Zeyada, H. M. \& El-Nahass, M. M. Electrical properties and dielectric relaxation of thermally evaporated zinc phthalocyanine thin films. Appl. Surf. Sci. 254, 1852-1858 (2008)

26. Senthilarasu, S., Hahn, Y. B. \& Lee, S.-H. Structural analysis of zinc phthalocyanine (ZnPc) thin films: X-ray diffraction study. J. Appl. Phys. 102, 043512 (2007).

27. Yu, W.-L., Pei, J., Cao, Y. \& Huang, W. Hole-injection enhancement by copper phthalocyanine (CuPc) in blue polymer light-emitting diodes. J. Appl. Phys. 89, 2343-2350 (2001).

28. Li, L., Guan, M., Cao, G., Li, Y. \& Zeng, Y. Low operating-voltage and high power-efficiency OLED employing MoO 3-doped CUPc as hole injection layer. Displays 33, 17-20 (2012).

29. Tianyong, Z. \& Chunlong, Z. Properties of copper phthalocyanine blue (C.) Pigment Blue 15:3) treated with poly(ethylene glycol)s. Dye. Pigment. 35, 123-130 (1997).

30. Ghani, F., Kristen, J. \& Riegler, H. Solubility properties of unsubstituted metal phthalocyanines in different types of solvents. J. Chem. Eng. Data 57, 439-449 (2012). 